\title{
Association between the morphometric parameters of placenta and umbilical cord with the birth weight
}

\author{
Nazeefa $\mathrm{HMFJ}^{1}$, Thirukumar $\mathrm{M}^{2}$, Thayabaran $\mathrm{M}^{1}$ \\ ${ }^{\prime}$ Department of Human Biology, Faculty of Health-Care Sciences, Eastern University, \\ Sri Lanka \\ ${ }^{2}$ Department of Clinical Sciences, Faculty of Health-Care Sciences, Eastern University, \\ Sri Lanka
}

Running title: Association of placenta and birth weight

\begin{abstract}
Objectives: Birth weight is a vital parameter used to predict the future growth and development of a new born. The survival of the foetus in utero depends on proper functions of the placenta. The study aims to express the relation between the birth weight and placental and umbilical cord morphometric parameters.
\end{abstract}

Materials and methods: The study was conducted on 418 singleton normal vaginal deliveries. The collected placentae were washed to remove the blood clots and the weight, thickness, and diameters of placentae and the length of umbilical cord were measured. Shape and margin of the placentae, location of umbilical cord insertion and number of cotyledons and the twist pattern of umbilical cord have been determined through careful examination. the birth weight of the foetus was obtained immediately after the delivery.
Results: The collected data has been analysed by multiple linear regression test using $\mathrm{R}$ software with $\mathrm{p}$ value $\leq$ 0.05 as the significance. The weight of the foetus exhibited positive significant linear relationship with thickness $(\mathrm{p}=0.001)$, weight $(\mathrm{p}=0.000)$ and Surface area $(\mathrm{p}=0.000)$ of the placental disk. The velamentous cord insertion showed $(p=0.019)$ negative relation with birth weight. The non-twist umbilical cord showed positive significant $(\mathrm{p}=0.04)$ association with birth weight. Other parameters were not significant with the birth weight.

Conclusion: Thickness, weight and surface area of the disk and non-twist umbilical cord increases the birth weight of the foetus. However, velamentous cord insertion related to reduction in the birth weight.

Keywords: Birth weight, thickness, weight, surface area, velamentous cord insertion

This is an open-access article distributed under the terms of the Creative Commons Attribution 4.0 International License, which permits unrestricted use, distribution and reproduction in any medium provided the original author and source are credited. 
Nazeefa HMFJ, Thirukumar M, Thayabaran M - Association between the morphometric parameters of placenta and umbilical cord with the birth weight

\section{Introduction}

Birth weight is a vital measurement to predict the future growth and wellbeing of the new born [1]. Birth weight of new born has been taken within first hours of life before any significant postnatal weight loss. Low birth weight babies are new born weighing less than $2,500 \mathrm{~g}$ and it is defined by World Health Organization (WHO) [2).

The placenta plays a crucial role in the development of foetus. It provides appropriate environment for the fetal growth in various aspects like nutrition, respiration, excretion of substances and protection [3]. Insufficiency of placental function leads to low birth weight, preterm birth and birth defects [4].

Placental morphometric parameters play different role in the functions of placenta. Standard placental measures include placental disk shape, diameter, surface area, disk thickness, weight, location of umbilical cord insertion site relative to the edge or margins of the placental disk, and placental weight in relation to birth weight [5].

Placental weight takes account of the different dimensions of lateral expansion of growth plate. They are measured by chorionic plate shape, the distance from the cord insertion site to the nearest chorionic plate margin and chorionic plate diameters [6]. Lateral growth expansion of the placental disk highlights the normal placental function, requires for the high capacitance and low resistance vascular system to combat the demand of the utero-placento-fetal unit [7].
Umbilical cord is the vital structure for the development, well-being, and survival of the fetus. However, it is vulnerable to kinking, compressions, traction, and torsion which may affect the perinatal outcome. The (clockwise) left and right (anti-clockwise) twisting pattern of the umbilical cord might play a minor role in altering the blood flow and determining the vasculature pattern of placenta [8].

The size, morphology and transferring capacity of the placenta and umbilical cord determines the prenatal growth of foetus to effect the birth weight. Therefore, evaluation of placenta after the birth would be an indicator to detect the growth restriction during the antenatal life and provide the opportunity for postnatal care [9].

The present study has been aimed to explore the relation between the morphometric parameters of the placenta and its umbilical cord and birth weight of foetus as a statistical model in the form of a formula.

\section{Materials and method}

The study was conducted on 418 placentae of consecutive deliveries from obstetrics and Gynaecology unit of Teaching Hospital, Batticaloa in three-month duration. The ethical clearance was granted by Ethics Review Committee of the Faculty of Health-Care Sciences, Eastern University, Sri Lanka under approval No: EUSL/FHCS/ERC/2017/30 to conduct the study. Informed written consent has been obtained from the 
Nazeefa HMFJ, Thirukumar M, Thayabaran M - Association between the morphometric parameters of placenta and umbilical cord with the birth weight

participants. The study included mothers with singleton normal vaginal deliveries of gestational age between 34 - 40 weeks.

The participants without antenatal check-up, and with the history of chronic diseases, as well as the placentae with less than 34 weeks gestation were excluded from the study. The data of maternal characters, morphometry of placenta and foetal parameters were recorded in a predesigned record sheet.

The placentae were collected immediately after separation from the new born. They were washed under running tap water to remove the blood clots. Then the membranes were trimmed and dried with the help of blotting paper before the doing thorough examination.

\section{Assessment of placental morphometry}

The shape and margin of the placentae, location of the insertion of umbilical cord and number of cotyledons were determined through careful examination.

The thickness of placentae has been measured in millimetres by inserting a fine needle at the centre of the placenta.

The weight of the placentae has been determined in nearest grams by using foetal weighing scale.

The diameter of placentae was measured using calibrated metallic scale in centimetres to calculate the surface area. The maximum two diameter was taken at right angle to each other. The means of the two measurements was considered as the diameter of placenta. The surface area was determined using the formula $\pi \mathrm{r} 2$ (the radius $r$ was calculated using the half of diameters (d) according to the formula $-r=d / 2$ ).

\section{Assessment of umbilical cord morphometry}

The length of umbilical cord was measured using calibrated metallic scale in centimetres excluding the first $5 \mathrm{~cm}$ from the infant's abdomen.

The (clockwise) left and right (anticlockwise) twisting pattern of the umbilical cord has been via thorough examination.

\section{Assessment of new born baby's parameters}

The birth weight of the new born baby was measured in nearest grams using foetal weighing scale, immediately after the delivery.

The statistical analysis has been carried out using R Software, version 4.0.0. The multiple linear regression model was done. The dependent variable is birth weight of the fetus and the independent variables are the shape of the placental disk, location of the umbilical cord insertion, Thickness of the placental disk, margins of the disk, Weight, Surface area, numbers of cotyledons, umbilical cord length and Twisting pattern of umbilical cord. 
Nazeefa HMFJ, Thirukumar M, Thayabaran M - Association between the morphometric parameters of placenta and umbilical cord with the birth weight

Among the independent variables shape of the placental disk, location of the umbilical cord insertion, margins of the disk, and Twist patterns are categorical variable.

In multiple linear regression, the R2 represents the correlation coefficient between the values. The value of $\mathrm{R}$ will always be positive and will range from zero to one [10]. The analysis was done using R software with the $95 \%$ confidence interval and $p$ value $\leq 0.05$ as the significance level to predict a model to find out the correlation between birth weight of the fetus and morphometric parameters of placenta and umbilical cord.

\section{Results}

The analysis includes morphometric parameters of 418 placentae. The thickness $(p=0.001)$, Weight $(p=0.000)$ and Surface area $(\mathrm{p}=0.000)$ of the placental disk were continuous independent variables, showed the significant $(p<0.05)$ linear relationship with the birth weight (intercept). It showed the linear relationship among the categorical independent variables for instance, the location of the umbilical cord insertion and twist pattern were significant. Among the location of the umbilical cord insertion only the velamentous type $(\mathrm{p}=0.019)$ was significant when compared to Central type of cord insertion $(\mathrm{P}<0.05)$. The velamentous type reduced the birth weight of the fetus by -0.248 when compared to Central type. Similarly, among Twist pattern, only the umbilical cord non-twist pattern $(\mathrm{p}=0.042)$ was significant when compared to left twist $(p<0.05)$. The umbilical cord with nontwist pattern increased the birth weight of the fetus by 0.322 . Other parameters were not significant with birth weight according to the findings.

The overall model was significant $(\mathrm{p}<0.05)$ with the adjusted (R2) R squared $49.26 \%$. The residual analysis was done to confirm the further model significance. The variation inflation factor for all independent variable was less than two and it was in acceptable level. The homogeneity of variance was tested by Breusch-Pagan test. The results showed that there was homogeneity of variance $(\mathrm{p}=0.8445 / \mathrm{p}>0.05)$. Auto correlation among the independent variables was tested by Durbin-Watson test and it showed that there was no autocorrelation $(\mathrm{p}=0.2448 / \mathrm{p}>0.05)$. The normality of residual was tested by Anderson-Darling test. The results showed that the residual was normally distributed $(p=0.089 / p>0.05)$. Since four residual analysis test showed acceptable result. We would able to confirm that the overall model was significant.

The model expressed as follow:

Birth Weight of fetus $=0.99+$ 0.126 (Thickness of placenta) + 0.0021 (Weight $)+0.0029$ (Surface area $)$ - 0.248(Velamentous cord insertion) + 0.322 (non-twist pattern of umbilical cord) 
Nazeefa HMFJ, Thirukumar M, Thayabaran M - Association between the morphometric parameters of placenta and umbilical cord with the birth weight

Table I: Multiple linear regression analysis of morphometric parameters of placenta and umbilical cord in association with Birth Weight

\begin{tabular}{|c|c|c|c|}
\hline Morphometry & Coefficient value & $p$ Value & \\
\hline Birth weight (Intercept) & 0.9904160 & 0.000 & $* * *$ \\
\hline \multicolumn{4}{|l|}{ Shape of placentae } \\
\hline Oval & -0.0512188 & 0.2304 & \\
\hline Irregular & -0.0208177 & 0.6746 & \\
\hline \multicolumn{4}{|l|}{ Location of Cord Insertion } \\
\hline Paracentral & 0.0082403 & 0.8493 & \\
\hline Eccentric & -0.0403800 & 0.4182 & \\
\hline Marginal & -0.0987913 & 0.1205 & \\
\hline Velamentous & -0.2478008 & 0.0195 & $*$ \\
\hline Thickness & 0.1258761 & 0.0017 & $* *$ \\
\hline \multicolumn{4}{|l|}{ Margins of placentae } \\
\hline Irregular & -0.0427965 & 0.2240 & \\
\hline Weight of placentae & 0.0020508 & 0.000 & $* * *$ \\
\hline Surface area of placentae & 0.0028627 & 0.000 & $* * *$ \\
\hline Number of cotyledons & -0.0075555 & 0.0620 & \\
\hline Cord length & 0.0021164 & 0.1336 & \\
\hline \multicolumn{4}{|l|}{ Cord twist } \\
\hline Anti-clock (Right) twist & 0.0605055 & 0.0717 & \\
\hline Non-twist & 0.3217109 & 0.0420 & $*$ \\
\hline
\end{tabular}

\section{Discussion}

Birth weight of the foetus increases by $1.98 \mathrm{~g}$ for every one-gram increase of placental weight [11]. It is clear that placental weight determines the fetal growth and birth weight [12]. In addition, placental growth significantly associates with early childhood growth in full-term infants [13]. Increase in nutrient exchange reflects on the arborisation of the villous and thickness of the chorionic disk [6]. Therefore, reduction in the surface area and weight than normal leads to slower rate of growth in foetus [9] and increase the risk of developing fetal growth restriction [14]. The model derived from our research findings expresses the direct relationship between the birth weight and the thickness, weight and surface area of placental disk along with previous findings.

Morphometrically, the location of the insertion of umbilical cord to the placenta has been considered as central, eccentric, marginal, and velamentous (membranous) insertions. Among them, marginal cord insertion and 
Nazeefa HMFJ, Thirukumar M, Thayabaran M - Association between the morphometric parameters of placenta and umbilical cord with the birth weight

velamentous cord insertions are considered as abnormal insertion to the placenta. Further, Ismail and colleagues, have identified that the abnormal placental cord insertion leads to low birth weight [15]. It has also been supported by several other research studies. One of such is that the usage of tobacco increases the prevalence of velamentous cord insertion and fetal growth retardation [16]. In addition, velamentous cord insertion increases the risk of birthweight difference in monochorionic twins [17]. Researches also shown that the occurrence of velamentous cord insertion is higher in the in vitro fertilization technique, maternal smoking, preterm delivery, single umbilical artery, lobed placenta and placenta previa [18]. Supportive to the above research findings, the model that we have derived, proves that velamentous cord insertion causes the reduction of birth weight and relates to adverse perinatal outcomes. Therefore, we could recommend that Routine examination of the placental cord insertion site need to be considered in future to avoid adverse perinatal consequences.

Considering the twisting pattern of the umbilical cord, hyper twist and nontwist umbilical cords are considered as abnormal twist. The frequency of occurrence is $4 \%-5 \%$ and it is associate with gestational diabetes and pre-eclampsia [20]. Non twist pattern is more prevalent in women carrying multiples due to the limited uterine environment. Also the non-twist pattern indicates the limited movement of foetus within the uterus. It was evidenced that; Hyper twist cord is associated with lower birth weight than the non-twist cord [21]. However, research evidences shown that the birth weight of the foetus is less likely affected by any of twist pattern [10]. In accordance to the previous research evidences, our findings suggest that non-twist cords are positively associate with birth weight despite of its adverse fetal outcome.

\section{Conclusion}

Placental morphometry: thickness, weight and surface area of the disk have exhibited significant and positive relationship with birth weight of the foetus. However, the velamentous cord insertion has the negative impact on the determinants of birth weight. Even though, the prevalence of the non-twist umbilical cord is minimal occurrence, it has a positive association with birth weight.

\section{Conflict of interest}

None declared.

\section{Acknowledgement}

Authors would appreciate the pregnant mothers who participated in the study and the staff of labour ward of Obstetrics and Gynaecology unit, Teaching Hospital, Batticaloa for their tremendous support during the study. Authors expresses their gratitude to $\mathrm{Mr}$ S Santharooban for his valuable advice and guidance on the statistical analysis. 
Nazeefa HMFJ, Thirukumar M, Thayabaran M - Association between the morphometric parameters of placenta and umbilical cord with the birth weight

\section{Correspondence -}

Dr. H.M.F.J. Nazeefa,

Department of Human Biology,

Faculty of Health-Care Sciences,

Eastern University, Sri Lanka,

No.50, New Kalmunai Road,

Batticaloa.

E-mail: nazeefaj@esn.ac.lk

Tel: +94 770661050

\section{References}

1. S. K. Sebayang, M. J. Dibley, P. J. Kelly, A. V. Shankar, and A. H. Shankar, (2012. "Determinants of low birthweight, small-for gestational-age and preterm birth in Lombok, Indonesia: analyses of the birthweight cohort of the SUMMIT trial," Tropical Medicine \& International Health: 17 (8): pp. 938-950.

2. Brown, L. D., \& Hay, W. W., Jr (2016). Impact of placental insufficiency on fetal skeletal muscle growth. Molecular and cellular endocrinology, 435, 69-77. https://doi.org/10.1016/j.mce.2016. 03.017

3. Burton G.J. (2001) Placental Functional Morphology. In: Augustin H.G., Rogers P.A.W., Iruela-Arispe M.L., Smith S.K. (eds) Vascular Morphogenesis in the Female Reproductive System. Cardiovascular Molecular Morphogenesis. Birkhäuser, Boston, MA. https://doi.org/10.1007/978-14612-0213-4_15

4. V. Kowsalya, R. Vijayakumar, G. Valli, K.P. Bharath, R. Srikumar, C. Kishor Kumar, I. Gayathri Fathima and N. Vanajashi, (2013).

Morphometry Examination of Placenta in Birth Weight of FullTerm Newborns in Puducherry, India. Pakistan Journal of Biological Sciences, 16: 895-897. http://dx.doi.org/10.3923/pjbs.2013 .895 .897

5. T. Yee Khong, Eoghan E. Mooney, Ilana Ariel, Nathalie C. M. Balmus, Theonia K. Boyd, Marie-Anne Brundler, Hayley Derricott, Margaret J. Evans, Ona M. FayePetersen, John E. Gillan, Alex E. P. Heazell, Debra S. Heller, Suzanne M. Jacques, Sarah Keating, Peter Kelehan, Ann Maes, Eileen M. McKay, Terry K. Morgan, Peter G. J. Nikkels, W. Tony Parks, Raymond W. Redline, Irene Scheimberg, Mirthe H. Schoots, Neil J. Sebire, Albert Timmer, Gitta Turowski, J. Patrick van der Voorn, Ineke van Lijnschoten, and Sanne J. Gordijn (2016). Sampling and Definitions of Placental Lesions: Amsterdam Placental Workshop Group Consensus Statement. Archives of Pathology \& Laboratory Medicine: 140 (7): pp. 698-713. https://doi.org/10.5858/arpa.20150225-CC

6. Salafia CM, Maas E, Thorp JM, Eucker B, Pezzullo JC, Savitz DA, 
Nazeefa HMFJ, Thirukumar M, Thayabaran M - Association between the morphometric parameters of placenta and umbilical cord with the birth weight

(2005). Measures of placental growth in relation to birth weight and gestational age. American Journal of Epidemiology: 162(10):991-998. doi:10.1093/aje/kwi305.

7. Helen Kay, D. Michael Nelson, Yuping Wang, (2011). The Placenta: From Development to Disease. $1^{\text {st }}$ edition. Blackwell Publishing Ltd: pp255.

8. Suresh Narayanan, Mrudula Munibala, (2018). Umbilical cord twist - Does it play a role in placental morphology? European Journal of anatomy: 22 (6): pp.483-488.

9. Salavati N, Smies M, Ganzevoort W, Charles AK, Erwich JJ, Plösch $\mathrm{T}$ and Gordijn SJ (2019). The Possible Role of Placental Morphometry in the Detection of Fetal Growth Restriction. Frontiers in Physiology. 9:1884. doi: 10.3389/fphys.2018.01884. https://doi.org/10.3389/fphys.2018. 01884

10. James, Gareth, Daniela Witten, Trevor Hastie, and Robert Tibshirani. (2014). An Introduction to Statistical Learning: With Applications in R. Springer Publishing Company, Incorporated. doi: 10.1007/978-1-4614-7138-7.

11. Sanin L, H, López S, R, Olivares E, T, Terrazas M, C, Silva M, A, R, Carrillo M, L, (2001). Relation between Birth Weight and Placenta Weight. Biology of the neonate: 80
(2):113-117. doi: 10.1159/000047 129

12. Roland, M. C., Friis, C. M., Voldner, N., Godang, K., Bollerslev, J., Haugen, G., \& Henriksen, T. (2012). Fetal growth versus birthweight: the role of placenta versus other determinants. PloS one, 7(6), e39324.

https://doi.org/10.1371/journal.pon e. 0039324

13. Ashraf T. Soliman, Mohamed Eldabbagh, Wail Saleem, Khaled Zahredin, Emad Shatla, Ashraf Adel, (2013). Placental weight: Relation to maternal weight and growth parameters of full-term babies at birth and during childhood, Journal of Tropical Pediatrics: 59 (5): Pages 358-364, https://doi.org/10.1093/tropej/fmt0 30

14. Hong-Jiao Liu, Peng-Cheng Liu, Jing Hua, Yan Zhao \& Jia Cao (2019). Placental weight and size in relation to fetal growth restriction: a case-control study, The Journal of Maternal-Fetal \& Neonatal Medicine. doi: 10.1080/14767058.2019.1636371

15. Ismail, K.I., Hannigan, A., O'Donoghue, and K., Cotter, A, (2017). Abnormal placental cord insertion and adverse pregnancy outcomes: a systematic review and meta-analysis. Syst Rev 6, 242. https://doi.org/10.1186/s13643017-0641-1

16. Sophie Brouillet, Anaïs Dufour, 
Nazeefa HMFJ, Thirukumar M, Thayabaran M - Association between the morphometric parameters of placenta and umbilical cord with the birth weight

Fabien Prot, Jean-Jacques Feige, Véronique Equy, Nadia Alfaidy, Pierre Gillois, and Pascale Hoffmann, (2014). Influence of the Umbilical Cord Insertion Site on the Optimal Individual Birth Weight Achievement. Biomed Research International. 2014 (341251). https://doi.org/10.1155/2014/34125 1

17. Costa-Castro T, De Villiers S, Montenegro N, Severo M, Oepkes D, Matias A, Lopriore E, (2013). Velamentous cord insertion in monochorionic twins with or without twin-twin transfusion syndrome: Does it matter? Placenta; 34(11):1053-1058. doi:10.1016/j.placenta.2013.08.00 9

18. Suzuki, S., \& Kato, M. (2015). Clinical Significance of Pregnancies Complicated by velamentous Umbilical Cord Insertion Associated with Other Umbilical Cord/Placental Abnormalities. Journal of clinical medicine research, 7(11), 853-856. https://doi.org/10.14740/jocmr231 0w

19. Kalish, Robin \& Hunter, Tiffany \& Sharma, Geeta \& Baergen, Rebecca. (2003). Clinical significance of the umbilical cord twist. American journal of obstetrics and gynecology. 189. 736-9. doi:10.1067/S00029378(03)00715-4.

20. Diwakar, R. K., Naik, M. M., \& Jindal, M. M. (2016). Umbilical cord coiling: case report and review of literature. $B J R$ case reports, 3(1), 20150152. https://doi.org/10.1259/bjrcr.20150 152

21. Jayshri Wakpnjar, Hemraj R Narkhede, Amol P Pawar, Pravin N Mhatre. (2016). Evaluation of association of abnormal umbilical cord coiling and perinatal outcome. International Journal of Contemporary Medical Research: 3(11):3305-3309. 